\title{
INVERSE PROBLEMS OF SYMBOLIC DYNAMICS
}

\author{
ALEXEI Ya. BELOV \\ Moscow Institute of Open Education \\ Aviatsionnyi per., 125167 Moscow, Russia \\ E-mail:kanel@mccme.ru \\ and \\ Shanghai University \\ 99 Shangda Road, BaoShan District, 200444 Shanghai, China \\ E-mail:belov@shu.edu.cn \\ GRIGORII V. KONDAKOV \\ Moscow Institute of Physics and Technology \\ 9, Institutski per., 141700 Dolgoprudny, Moscow Region, Russia \\ E-mail:kondakov@yandex.ru \\ IVAN V. MITROFANOV \\ Moscow State University \\ Leninskie Gory, 119992 Moscow, Russia \\ E-mail: vanessfortim@mail.ru
}

\begin{abstract}
This paper reviews some results regarding symbolic dynamics, correspondence between languages of dynamical systems and combinatorics. Sturmian sequences provide a pattern for investigation of one-dimensional systems, in particular interval exchange transformation. Rauzy graphs language can express many important combinatorial and some dynamical properties. In this case combinatorial properties are considered as being generated by a substitutional system, and dynamical properties are considered as criteria for a superword being generated by an interval exchange transformation. As a consequence, one can get a morphic word appearing in an interval exchange transformation such that the frequencies of the letters are algebraic numbers of an arbitrary degree.
\end{abstract}

2010 Mathematics Subject Classification: Primary 37B; Secondary 05E.

Key words and phrases: combinatorics of words, Sturmian sequence, interval exchange transformation, morphic sequence, symbolical dynamics, Weyl lemma, unipotent torus transformation, complexity function.

The paper is in final form and no version of it will be published elsewhere. 
Concerning multidimensional systems, our main result is the following. Let $P(n)$ be a polynomial, having an irrational coefficient of the highest degree. A word $w\left(w=\left(w_{n}\right), n \in \mathbb{Z}\right)$ consists of a sequence of the first binary numbers of $\{P(n)\}$, i.e. $w_{n}=[2\{P(n)\}]$. Denote the number of different subwords of $w$ of length $k$ by $T(k)$. We prove that there exists a polynomial $Q(k)$, depending only on the power of the polynomial $P$, such that $T(k)=Q(k)$ for sufficiently large $k$.

1. Introduction. The methods of symbolic dynamics are useful in the study of combinatorial properties of words, in some problems of number theory and in the theory of dynamical systems. Let $M$ be a compact metric space, $U \subset M$ be its open subspace, $f: M \rightarrow M$ be a homeomorphism and $x_{0} \in M$ be an initial point. It determines a sequence of points

$$
x_{0}, f\left(x_{0}\right), \ldots, f^{(n)}\left(x_{0}\right), \ldots
$$

To this sequence of iterations, one can associate an infinite binary word $W=\left\{W_{n}\right\}$ where

$$
W_{n}=\left\{\begin{array}{c}
a, f^{(n)}\left(x_{0}\right) \in U, \\
b, f^{(n)}\left(x_{0}\right) \notin U,
\end{array}\right.
$$

which is called the evolution of the point $x_{0}$. If $f$ is invertible then $n \in \mathbb{Z}$, otherwise $n \in \mathbb{N}$. Symbolic dynamics investigates the interrelation between the properties of the dynamical system $(M, f)$ and the combinatorial properties of the word $W$. For words over alphabets which comprise more symbols, several characteristic sets should be considered: $U_{1}, \ldots, U_{n}$. The technical notions regarding combinatorics of words can be found in section 5.1 .

The direct problem of symbolic dynamics consists in the description of the properties of the word $W$, based on the information about the dynamic system. The inverse problem consists in the description of $(M, f, U, x)$, based on the information about $W$.

We shall point out some facts, which are folklore.

The minimality of the dynamical system corresponds to the uniform recurrence property (see section 5.1).

The uniqueness of invariant measure corresponds to the following property. Let $u$ be a subword of a uniformly recurrent (u.r.) word $W$. For any subword $u \sqsubset W$ let us suppose that the upper density of occurrence coincides with the lower density. The invariant measure is then unique.

In what cases is $M$ a torus and $f: M \rightarrow M$ its rotation? This means that this dynamical system has a discrete spectrum. Let $W$ be a superword, obtained by this dynamics. Let $T$ be the shift operator. Then the mismatch function between $W$ and $T^{n}(W)$ satisfies the following conditions:

1. There exists a sequence $\left\{n_{i}\right\}$ such that $\rho\left(T^{n_{i}}(W), W\right) \rightarrow 0$.

2. There exist co-prime arbitrarily large $n_{i}, n_{j}$ from this sequence.

We shall analyze these problems. We start from general constructions, regarding torus rotation questions, uniqueness of the invariant measure, minimality of the dynamical system.

The famous Sturmian sequences and some of their generalizations present a situation of "combinatorial paradise". It provides patterns for further investigation. Using the 
language of Rauzy graphs we shall formulate criteria for a superword being generated by an interval exchange transformation. Note that any billiard word with rational angles can be obtained via such transformations. (The number of directions of the ball is finite and the position of the ball on the side together with its direction provides a phase point, and the phase space is the union of some intervals.) On the other hand, using the language of Rauzy schemes (obtained from Rauzy graphs by replacing the maximal sequences of vertices of ingoing and outgoing degree 1 by arcs) we get criteria for the superword to be morphic.

Concerning shifts of a multidimensional torus, there is a beautiful theory of Rauzy fractals. For more complicated systems one needs other patterns for investigation rather than those provided by Sturmian sequences.

The rest of this paper is devoted to dynamic systems connected with unipotent transformations of a torus. These subject was considered in [3]. The issues related to the study of sequences, obtained by taking a fractional part of the values of a polynomial at integer points lead to the investigation of such dynamic systems. These problems play an important role in the theory of numbers, the theory of information transfer and some other branches [38, 27, 30. Note that unipotent transformations of $\mathbb{T}^{2}$ have the same relation to a circle shift as billiards with arbitrary angles to interval exchange transformations. The sequences appearing in such billiards are analyzed in [16.

The inverse problems of symbolic dynamics related to unipotent transformations of a torus were studied in [10] (unfortunately it was published only in Russian).

We have to point out that the results in [3] were obtained independently of [10]. Let $Q(X)$ be a real polynomial of degree $d \geq 1$ where the coefficient of $X^{d}$ is irrational. Define the difference operator $\Delta u_{n}=u_{n+1}-u_{n}$ and its iterates $\Delta^{2}=\Delta \circ \Delta, \cdots, \Delta^{d}=\Delta \circ \Delta^{d-1}$. The authors establish that the sequence $\left(\Delta^{d}\lfloor Q(n)\rfloor\right)_{n \geq 0}$ takes its values in a $2^{d}$-element alphabet and that the number $p_{d}(n)$ of its subwords of length $n$ is

$$
p_{d}(n)=\frac{1}{V(0,1, \cdots, d-1)} \sum_{0 \leq k_{1}<\cdots<k_{d} \leq n+d-1} V\left(k_{d}, \cdots, k_{1}\right),
$$

where

$$
V\left(k_{d}, \cdots, k_{1}\right)=\prod_{1 \leq i<j \leq d}\left(k_{j}-k_{i}\right),
$$

is the Vandermonde determinant. In particular, $p(n)$ depends only on the degree $d$ of the polynomial $Q$ provided that the coefficient of $X^{d}$ is irrational, $p_{2}(n)=(n+1)(n+2)(n+$ $3) / 6$.

In this situation one has to count the number of parts of the torus division by images of a hyperplane. And the proof that points in different regions have different evolutions can be done just by dimension induction because this system provides more information; no theory of quasi-invariant sets and factor dynamics is required.

Let $P(n)$ be a polynomial having an irrational coefficient of the highest degree. The word $w\left(w=\left(w_{n}\right), n \in \mathbb{Z}\right)$ consists of a sequence of first binary numbers of $\{P(n)\}$, i.e. $w_{n}=[2\{P(n)\}]$. Denote by $T(k)$ the number of different subwords of $w$ of length $k$.

The main theorem of this section is as follows: 
TheOREM 1.1. There exists a polynomial $Q(k)$, depending only on the degree of the polynomial $P$, such that $T(k)=Q(k)$ for all sufficiently large $k$.

2. Sturmian sequences and their generalizations. The problems (both direct and inverse) related to the rotation of a circle lead to a class of words which are called Sturmian words. Sturmian words are infinite words over a binary alphabet which contain exactly $n+1$ different subwords (factors) of length $n$ for any $n \geq 1$.

Sturmian words provide an example of correspondence between the language of dynamical system and the combinatorial properties of superwords. We shall formulate a classical result:

THEOREM 2.1 (Equivalence theorem [35], 32]). Let $W$ be an infinite recurrent word over the binary alphabet $A=\{a, b\}$. The following conditions are equivalent:

1. The word $W$ is a Sturmian word, i.e., for any $n \geq 1$, the number of different subwords of length $n$ that occur in $W$ is equal to $T_{n}(W)=n+1$.

2. The word is not periodic and is balanced, i.e., any two subwords $u, v \subset W$ of the same length satisfy the inequality $\left.|| v\right|_{a}-|u|_{a} \mid \leq 1$, where $|w|_{a}$ denotes the number of occurrences of the symbol a in the word $w$.

3. The word $W=\left(W_{n}\right)$ is a mechanical word with irrational $\alpha$, which means that there exist an irrational $\alpha, x_{0} \in[0,1]$, and an interval $U \subset \mathbb{S}^{1},|U|=\alpha$, such that the following condition holds:

$$
W_{n}=\left\{\begin{array}{cc}
a, & T_{\alpha}^{n}\left(x_{0}\right) \in U, \\
b, & T_{\alpha}^{n}\left(x_{0}\right) \notin U .
\end{array}\right.
$$

4. The word $W$ can be obtained as a limit of the sequence of finite words $\left\{w_{i}\right\}_{i=1}^{\infty}$, such that $w_{i+1}$ can be obtained from $w_{i}$ via a substitution of the following type $a^{k_{i}} b \rightarrow b, a^{k_{i}+1} b \rightarrow a$ or $b^{k_{i}} a \rightarrow a, b^{k_{i}+1} a \rightarrow b$.

The sequence of these substitutions is periodic if and only if $\alpha$ is a quadratic irrational.

Sturmian words can be considered as a theoretical "paradise" and a pattern for further investigations. There are several different ways of generalizing Sturmian words.

First, one can consider balanced words over an arbitrary alphabet. Balanced nonperiodic words over an $n$-letter alphabet were described in [24] and later in [26]. In [17], a dynamical system that generates an arbitrary nonperiodic balanced word was constructed.

Secondly, a generalization may be formulated in terms of the complexity function. The complexity function $T_{W}(n)$ counts the number of different subwords of length $n$ in the word $W$. Sturmian words satisfy the relation $T_{W}(n+1)-T_{W}(n)=1$ for any $n \geq 1$. Some natural generalizations of Sturmian words are words with minimal growth, i.e., words over a finite alphabet that satisfy $T_{W}(n+1)-T_{W}(n)=1$ for any $n \geq k$, where $k$ is a positive integer. Such words were described in terms of rotations of a circle in [18. Note also that words whose growth function satisfies $\lim _{n \rightarrow \infty} T(n) / n=1$ were studied in [1]. 
The words with complexity function $T_{W}(n)=2 n+1$ were studied by P. Arnoux and G. Rauzy ([4, 41, 39]), the words with growth function $T_{W}(n)=2 n+1$ were analysed by G. Rote 42. The consideration of the general case of words with a linear complexity function involves the study of words generated by interval exchange transformations. The problem of description of such words was posed by Rauzy 39. The words with linear growth of the number of subwords were studied by V. Berthé, S. Ferenczi, Luca Q. Zamboni ([21], 13]). They investigated combinatorial sequences related to interval exchange transformations. See also works of P. Baláži, Z. Masáková, E. Pelantová ([5], [6], [7]).

The description of Rauzy's program of investigation of dynamical systems can be found in 13$]$.

3. Interval exchange transformations. Sturmian sequences can be obtained via rotations of the unit circle. Interval exchange transformations generalize circle rotations. G. Rauzy posed the question on the description of such words obtained by interval exchange transformations [39].

S. Ferenci and L.Zamboni [22] obtained the criteria for words generated by interval exchange transformations with the following condition: the trajectory of every break point does not get on any break point. In this case obviously the complexity function of the word is equal to $T(n)=(k-1) n+1$. In fact, this is the answer to the Rauzy question.

In [28, 9] the words generated by a general piecewise-continuous transformation of the interval were studied. This approach is quite different. The answer to this question is given in terms of the evolution of the labeled Rauzy graphs of the word $W$. The Rauzy graph of order $k$ (the $k$-graph) of the word $W$ is the directed graph whose vertices biuniquely correspond to the factors of length $k$ of the word $W$ and the vertex $A$ is connected to the vertex $B$ by a directed arc if and only if $W$ has a factor of length $k+1$ such that its first $k$ letters compose the subword that corresponds to $A$ and the last $k$ symbols compose the subword that corresponds to $B$. By the follower of the directed $k$-graph $G$ we understand the directed graph $\operatorname{Fol}(G)$ constructed as follows: the vertices of the graph $\operatorname{Fol}(G)$ are in one-to-one correspondence with the arcs of the graph $G$ and there exists an arc from the vertex $A$ to the vertex $B$ if and only if the head of the arc $A$ in the graph $G$ is at the notch end of $B$. The $(k+1)$-graph is a subgraph of the follower of the $k$-graph; it results from the latter by removing some arcs. Vertices which are tails of (or heads of) at least two arcs correspond to special factors; vertices which are heads and tails of more than one arc correspond to bispecial factors. The sequence of the Rauzy $k$-graphs constitutes the evolution of the Rauzy graphs of the word $W$. The Rauzy graph is said to be labeled if its arcs are assigned the letters $l$ and $r$ and some of its vertices (perhaps, none of them) are assigned the symbol "-". The follower of the labeled Rauzy graph is the directed graph which is the follower of the latter (considered as a Rauzy graph with the labeling neglected) and whose arcs are labeled according to the following rule:

1. Arcs that enter a branching vertex should be labeled by the same symbols as the arcs that enter any left successor of this vertex; 
2. Arcs that go out of a branching vertex should be labeled by the same symbols as the arcs that go out of any right successor of this vertex;

3. If a vertex is labeled by the symbol "_", then all its right successors should also be labeled by the symbol "--".

The evolution is said to be correct if, for all $k \geq 1$, the following conditions hold when passing from the $k$-graph $G_{k}$ to the $(k+1)$-graph $G_{k+1}$ :

1. Each vertex is incident to at most two incoming and outgoing arcs;

2. If the graph contains no vertices corresponding to bispecial factors, then $G_{n+1}$ coincides with the follower $D\left(G_{n}\right)$;

3. If the vertex that corresponds to a bispecial factor is not labeled by the symbol "-", then the arcs that correspond to forbidden words are chosen among the pairs $l r$ and $r l$;

4. If the vertex is labeled by the symbol "--", then the arcs to be deleted should be chosen among the pairs $l l$ or $r r$.

The evolution is said to be asymptotically correct if this condition is valid for all $k$ from a certain $k=K$ on. The oriented evolution of the graphs means that there are no vertices labeled by the symbol "-".

THEOREM 3.1 (9, 28, $)$. A uniformly recurrent word $W$ :

1. is generated by an interval exchange transformation if and only if the word is provided with the asymptotically correct evolution of the labeled Rauzy graphs;

2. is generated by an orientation-preserving interval exchange transformation if and only if the word is provided with the asymptotically correct oriented evolution of the labeled Rauzy graphs.

The proof of this theorem consists of two stages. First one proves that these conditions are sufficient for the word to be generated by a piecewise-continuous interval transformation. And the second step is to prove that the sets of uniformly recurrent words generated by the piecewise-continuous interval transformations and by the interval exchange transformation are equivalent. In order to do it, some invariant measure is introduced which provides a metric: the measure of a line segment is its length.

4. Substitutional sequences. An important class of sequences are the so-called substitutional sequences. These sequences are invariant under substitution. We refer to the paper 34]. The Fibonacci word is such an example. Let us consider the substitution $\phi: 0 \rightarrow 001,1 \rightarrow 01$. From the symbol 0 one can get the Fibonacci word as the superword $\phi^{\infty}(0)$. It is Sturmian. The Tribonacci word $\tau^{\infty}(0)=01020100102010$ can be generated by the substitution $\tau(0)=01, \tau(1)=02, \tau(2)=0$.

In fact, in [40] G. Rauzy showed that the Tribonacci minimal subshift (the shift orbit closure of t) is a natural coding of a rotation on the 2-dimensional torus $\mathbb{T}^{2}$; i.e., is measure-theoretically conjugate to an exchange of three fractal domains on a compact set in $\mathbb{R}^{2}$. Each domain is translated by the same vector modulo a lattice. 
This is one of the most impressive results. It provides a description of two dimensional spaces. The symbols correspond to the division of $\mathbb{T}^{2}$ into fractals, called Rauzy fractals. The theory of Rauzy fractals was generalized to the so-called Pisot substitutions.

This type of dynamical systems provides rich structures with a nice picture in the multidimensional case. We have a correspondence between the languages of arithmetic, dynamical systems and combinatorics.

In other multidimensional systems one can get some information. The complexity of sequences related to multidimensional systems was studied in [2].

4.1. Language of substitutions and Rauzy graphs. Consider condition (4) in Theorem 2.1. A Sturmian sequence can be obtained as a limit of very special substitutions. If it is invariant under some substitution, then the rotation number is a quadratic irrationality. A similar fact is known for any rotation of the circle. However, there exist substitutions such that the eigenvalues of the corresponding matrices are algebraic numbers of an arbitrary degree.

The language of Rauzy graphs provides a bridge between combinatorial and topological properties in problems regarding interval exchange transformations. The technique of Rauzy graphs is an important tool in combinatorics of words.

The Rauzy graph $G_{k}$ of a Sturmian sequence has one incoming and one outgoing branching vertex. When they coincide, (a bispecial word appears) $G_{k+1}$ can be obtained via choosing one of two possibilities, according to Theorem 3.1. This choice corresponds to the decomposition of $\alpha$ in chain fraction. If this choice is made in a periodic way, $\alpha$ is a quadratic irrational.

This fact can be generalized. Let $W$ be a u.r. word. Suppose that the behavior of Rauzy graphs is periodic in the same sense, then $W$ is equivalent to a superword invariant under some substitution. In order to formulate a theorem one should define what "periodicity of events" in Rauzy graphs means.

A Rauzy scheme of $W$ is a sequence of graphs $\left\{\Gamma_{i}\right\}$ such that every vertex of $\Gamma_{i}$ is either an outgoing or an incoming branching vertex of some order, and corresponds to a subword of $W . \Gamma_{i+1}$ can be obtained from $\Gamma_{i}$ via exchanging some paths of length 2 passed through some vertex via arrows and deleting vertices which are not endpoints of new arrows. A Rauzy scheme is periodic if there exists $k>0$ such that for all sufficiently large $i$ there is an isomorphism between $\Gamma_{i}$ and $\Gamma_{i+k}$ which fits with the transmission from $\Gamma_{i}$ to $\Gamma_{i+1}$.

THEOREM 4.1 (29]). A uniformly recurrent superword is equivalent to the image of some morphism of substitutional invariant sequences if and only if it has a periodic Rauzy scheme.

Proving that if $W$ has a periodic Rauzy scheme, then it is substitutional is not so difficult. The main obstacle is the opposite implication. A substitution can be "bad" in the sense that the image of a letter $a_{i}$ can have a "parasite" inclusion of the image of $a_{j}$. So this naive construction fails. We do not know an explicit construction.

Let us call weight of vertex of Rauzy scheme the length of the corresponding word. In order to prove the theorem, one needs to establish that if $W$ is a uniformly recurrent word 
stable under substitution, then the ratios of the weights of all Rauzy vertices are bounded. Then one can use J. Cassaigne's result [15] stating that if $W$ is uniformly recurrent and $\liminf T_{W}(n) / n<\infty$, then $\lim \sup T_{W}(n+1)-T_{W}(n)<\infty$. This follows from the fact that the number of vertices in Rauzy scheme is bounded and one gets the existence of a periodic Rauzy scheme from that. (The condition $\liminf T_{W}(n) / n<\infty$ for morphic uniformly recurrent words follows from results of Yu. Pritukin [36].)

The proof of the ratio boundedness is based on the following fact. If $W$ is a morphic uniformly recurrent word then there exists a constant $C(W)$ such that for any subword $W$ $u$ occurs in $v$ for any $v \sqsubset W,|v|>C(W) \cdot|u|$. In order to use it, one needs to construct a sequence of Rauzy schemes in such a way that paths incompatible by inclusion correspond to words with the same property.

This theorem implies the Vershik-Lifshits theorem [44, 45] of periodicity of Bratteli diagrams of Markov compacta corresponding to substitutional systems.

A Bratteli diagram $(V, E)$ is a countable collection $V$ of finite vertex sets, $V=\left\{V_{n}\right\}_{n=1}^{\infty}$ and a countable collection $E$ of finite edge sets $E=\left\{E_{n}\right\}_{n=1}^{\infty}$, along with functions $s: E_{n} \rightarrow V_{n-1}$ and $r: E_{n} \rightarrow V_{n}$ such that (i) $V_{0}=\left\{\nu_{0}\right\}$, (ii) $s: E_{n} \rightarrow V_{n-1}$ and $r: E_{n} \rightarrow V_{n}$ are onto for all $n$. We view $(V, E)$ as a directed graph where an edge $e \in E_{n}$ connects the source vertex $s(e) \in V_{n}$ to the range vertex $r(e) \in V_{n+l}$. Periodicity of Bratteli diagram means that for some $k$ there exists a pair of mappings from $V_{n}$ to $V_{n+k}$ and from $E_{n}$ to $E_{n+k}$ preserving functions $s$ and $r$. With the Bratteli diagram one can associate topological dynamics. Details can be found in [25].

The proof of the Vershik-Lifshits theorem uses a straightforward construction. Consider the image of $\varphi^{(n)}(a)=\left(\varphi^{(n-2)}\right)\left(\varphi^{(2)}(a)\right)$ for some letter $a$. It consists of blocks corresponding to the application of $\varphi^{(n-2)}$ to the letters of $\varphi^{(2)}(a)$ and also can be decomposed into the blocks corresponding to the application of $\varphi^{(n-1)}$ to the letters of $\varphi(a)$. The finite sets forming Bratteli diagrams, consist of sequences of pairs (block, its position in bigger block), they correspond to some subwords of $W$. On these sets the relations of being left and right neighbors inside a bigger block can be naturally defined. The first and last occurrence in the bigger block needs special attention, because the bigger block itself may have some position and can be preceded or followed by another bigger block. Details can be found in 44, 45. See also 31. The properties of adic transformations and interval exchange transformations and different invariant measures are studied in [14].

\section{Main constructions and definitions}

5.1. Complexity function, special factors, and uniformly recurrent words. In this section we define the basic notions of combinatorics of words. By $A$ we denote a finite alphabet, i.e., a nonempty set of elements (symbols). We use the notation $A^{+}$for the set of all finite sequences of symbols or words.

A finite word can always be uniquely represented in the form $w=w_{1} \cdots w_{n}$, where $w_{i} \in A, 1 \leq i \leq n$. The number $n$ is called the length of the word $w$; it is denoted by $|w|$.

The set $A^{+}$of all finite words over $A$ is a simple semigroup with concatenation as semigroup operation. 
If the element $\Lambda$ (the empty word) is included in the set of words, then this is actually the free monoid $A^{*}$ over $A$. By definition the length of the empty word is $|\Lambda|=0$.

A word $u$ is a subword (or factor) of a word $w$ if there exist words $p, q \in A^{+}$such that $w=p u q$.

Denote the set of all factors (both finite and infinite) of a word $W$ by $F(W)$. Two infinite words $W$ and $V$ over the alphabet $A$ are said to be equivalent if $F(W)=F(V)$. The beginning $w^{b}$ of length $b$ of the word $w$ can be defined in a natural way.

We say that the symbol $a \in A$ is a left (accordingly, right) extension of the factor $v$ if $a v$ (accordingly, $v a$ ) belongs to $F(W)$. A subword $v$ is called a left (accordingly, right) special factor if it possesses at least two left (right) extensions. A subword $v$ is said to be bispecial if it is both a left and right special factor at the same time. The number of different left (right) extensions of a subword is called the left (right) valence of this subword.

A word $W$ is said to be recurrent if any of its factors occurs infinitely many times (in the case of a doubly-infinite word, each factor occurs infinitely many times in both directions). A word $W$ is said to be uniformly recurrent (or u.r. word) if it is recurrent and, for each of its factor $v$, there exists a positive integer $N(v)$ such that, for any subword $u$ of length at least $N(v)$ of the word $W$, the factor $v$ occurs in $u$ as a subword.

Below we formulate several theorems about u.r. words, which will be needed later. The proof of these theorems can be found in the monograph 8 .

THEOREM 5.1. The following two properties of an infinite word $W$ are equivalent:

a) For any $k$ there exists $N(k)$ such that any segment of length $k$ of the word $W$ occurs in any segment of length $N(k)$ of the word $W$;

b) If all finite factors of a word $V$ are at the same time finite factors of a word $W$, then all finite factors of the word $W$ are also finite factors of the word $V$.

THEOREM 5.2. Let $W$ be an infinite word. Then there exists a uniformly recurrent word $\widehat{W}$ all of whose factors are factors of $W$.

One can consider the action of the shift operator $\tau$ on the set of infinite words. The Hamming distance between words $W_{1}$ and $W_{2}$ is the quantity $d\left(W_{1}, W_{2}\right)=\sum_{n \in \mathbb{Z}} \lambda_{n} 2^{-|n|}$, where $\lambda_{n}=0$ if the symbols at the $n$-th positions of the words coincide, and $\lambda_{n}=1$ otherwise.

An invariant subset is a subset of the set of all infinite words which is invariant under the action of $\tau$. A minimal closed invariant set, or briefly m.c.i.s., is a closed (with respect to the Hamming metric introduced above) invariant subset which is nonempty and contains no closed invariant subsets except itself and the empty subset.

THEOREM 5.3 (Properties of closed invariant sets). The following properties of a superword $W$ are equivalent:

1. $W$ is a uniformly recurrent word;

2. The closed orbit of $W$ is minimal and is a m.c.i.s. 
THEOREM 5.4. Let $W$ be a uniformly recurrent nonperiodic infinite word. Then

1. All the words which are equivalent to $W$ are u.r. words; the set of such words is uncountable;

2. There exist distinct u.r. words $W_{1} \neq W_{2}$ which are equivalent to the given word and can be written as $W_{1}=U V_{1}, W_{2}=U V_{2}$, where $U$ is a left-infinite word and $V_{1} \neq V_{2}$ are right-infinite words.

\section{Unipotent dynamics on a torus}

6.1. Essential evolution of points. Let $f: M \rightarrow M$ be a continuous map on the space $M$ and $U \subset M$ be a subset. The starting point $x$ determines a binary word $w$ describing the evolution as above: $w_{n}=1$, if $f^{(n)}(x) \in U$ and $w_{n}=0$ if $f^{(n)}(x) \notin U$. We assume that $U$ is an open set, $\operatorname{mes}(\partial U)=0$ and $M$ is a compact metric space.

Definition 1. $v_{x}^{b}$ denotes an evolution of length $b$ of point $x$. A finite word $v^{f}$ is said to be an essential finite evolution of a point $x^{*}$ if every neighborhood of a point $x^{*}$ contains an open set $V$ so that for all $x \in V$ the equality $v_{x}^{b}=v^{f}$ holds.

An infinite word $w$ is said to be an essential (infinite) evolution of a point $x^{*}$ if every initial subword is an essential finite evolution of a point $x^{*}$.

The word evolution will further mean essential evolution.

Proposition 6.1. Let $v$ be a finite word, then the set of points with fixed finite evolution is closed (i.e. contains all its limit points)

A similar statement holds for an infinite word $w$. (The intersection of any family of closed sets is closed.)

We shall not use the next proposition although it has its own interest:

Proposition 6.2. Let $(M, f, U, x)$ be a dynamical system without closed invariant subsets, and such that different points have different evolutions. Let $(\hat{M}, s, U, x)$ be the corresponding symbolical dynamical system, i.e. the set of all superwords with Tikhonov topology. Then $M$ is naturally isomorphic to the factor $\hat{M}$ by spaces consisting of sets of superwords which are an essential evolution of one point from $M$; this isomorphism induces natural isomorphisms of dynamical systems.

\subsection{Morphism of dynamics}

Definition 2. A morphism of two dynamics $G:\left(M_{1}, f_{1}\right) \rightarrow\left(M_{2}, f_{2}\right)$ is a continuous map such that the diagram

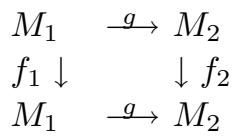

is commutative.

The notions of epimorphism, monomorphism and isomorphism are defined in the natural way. 
The factor-dynamics is naturally defined on the quotient topology if and only if $f$ permutes the equivalence classes of the map $f$. Note that the inverse image of a point under a morphism is closed.

Definition 3. A set $V$ is irreducible if its closure is not the inverse image of a closed set under any morphism (except monomorphism), and reducible otherwise.

THEOREM 6.3. Let a set $U$ be irreducible, then

1. Different points have different evolutions.

2. For any $\varepsilon>0$ there exists $N(\varepsilon)$ such that any two words of length $N(\varepsilon)$, corresponding to initial points with distance greater than $\varepsilon$ are different.

Proof. 1. Classes of points with the same evolution are closed and the map $f$ permutes them.

2. This point follows from the previous one by contradiction and a limit passage.

\subsection{Quasi-invariant sets}

Definition 4. A dynamics is said to be minimal if $M$ does not contain closed invariant sets apart from $M$ and $\varnothing$, and is said to be irreducible if it does not contain proper quasi-invariant sets.

Definition 5. A closed set $N$ is quasi-invariant if for any two points $A, B \in N$ and any convergent sequence $f^{\left(n_{i}\right)}(A) \rightarrow C$ such that $C \in N$ for $n_{i} \rightarrow \infty$ every limit point of the sequence $f^{\left(n_{i}\right)}(B)$ is in $N$.

Proposition 6.4.

1. Every closed invariant set is quasi-invariant.

2. A partition of quasi-invariant sets corresponds to each factor-dynamics and conversely.

3. The image of a quasi-invariant set is quasi-invariant.

4. The set of points with fixed evolution is quasi-invariant.

Definition 6. The $A-B$ cloud (or 0-cloud) with center $A$, generated by the point $B$ is the set of conditional limit points of $f^{\left(n_{i}\right)}(B)$ under the condition $f^{\left(n_{i}\right)}(A) \rightarrow A$.

The $A-B k$-cloud with center $A$, generated by the point $B$ is the closure of the union of sets of conditional limit points of $f^{\left(n_{i}\right)}(B)$ under the condition $f^{\left(n_{i}\right)}(A) \rightarrow A^{*}$ where $A^{*} \in A-B(k-1)$.

The $A-B \infty$-cloud with center $A$, generated by the point $B$ is the closure of the union of the sets of $A-B k$-clouds, $k \in N$.

Note that the $A-B k$-cloud is closed.

Proposition 6.5. a) The image of the $A-B k$-cloud under the $l$-th iteration is the $f^{(l)}(A)-f^{(l)}(B)$-cloud.

b) If $A_{n} \rightarrow A, B_{n} \rightarrow B$ then $\rho\left(A_{n}-B_{n}, A-B\right) \rightarrow 0$.

Denote the $A-B$-cloud by $L_{0}$, and denote by $L_{i+1}$ the closure of the union of all $A_{i}-B_{i}$-clouds, for which $A_{i}, B_{i} \in L_{i}$. Write $L_{A B}=\bigcup L_{i}$. The factorization generated by $L_{A B}$ is the weakest factorization that glues points $A$ and $B$. 
6.4. Unipotent dynamics on a torus. The problems connected with the study of the behavior of fractional parts of values of a polynomial at integer points are in fact the classical problems of symbolic dynamics. Let $P(n)$ be a polynomial of degree $m+1$ with irrational coefficient $a_{m+1}$ of the highest degree. Define a sequence of polynomials $P_{k}(n), k=0, \ldots, m$, in the following way:

$$
\left\{\begin{aligned}
P_{m}(n) & =P(n), \\
P_{m-1}(n) & =P_{m}(n+1)-P_{m}(n), \\
& \ldots \\
P_{i-1}(n) & =P_{i}(n+1)-P_{i}(n), \\
& \ldots
\end{aligned}\right.
$$

From these formulas it follows that $P_{0}(n)=n ! a_{m+1}$ is irrational. Put $\varepsilon=P_{0}(n), x_{i}(n)=$ $\left\{P_{i}(n)\right\}$ and $x_{i}^{\prime}(n)=x_{i}(n+1)$, then from (1) we obtain the following dynamical system:

$$
\left\{\begin{aligned}
x_{m}^{\prime} & =x_{m}+x_{m-1} \bmod 1 \\
x_{m-1}^{\prime} & =x_{m-1}+x_{m-2} \bmod 1 \\
& \cdots \\
x_{1}^{\prime} & =x_{1}+\varepsilon \bmod 1
\end{aligned}\right.
$$

where $\varepsilon$ is irrational as $\varepsilon=n ! a_{m+1}$. The condition $[2\{P(n)\}]=0$ turns into $0 \leq x_{m}(n)<$ $1 / 2$. Consequently the vector $\left(x_{1}^{\prime}, \ldots, x_{m}^{\prime}\right)$ is obtained from $\left(x_{1}, \ldots, x_{m}\right)$ by a unipotent transformation (corresponding to a linear transformation with unitary eigenvalues).

The images of the hyperplanes $x_{m}=0$ and $x_{m}=1 / 2$ divide the space into polyhedra. The same word of length $k$ corresponds to all points of the same polyhedron.

\subsection{Mismatch function}

Definition 7. The mismatch function $\rho$ of the words $w$ and $v$ is defined in the following way:

$$
\rho(i)= \begin{cases}0, & \text { if } w_{i}=v_{i}, \\ 1, & \text { if } w_{i} \neq v_{i},\end{cases}
$$

The density of mismatch of $\rho(w, v)$ of the words $w$ and $v$ is defined by the formula

$$
\rho(w, v)=\lim _{i \rightarrow \infty} \frac{\sum_{j=1}^{i} \rho(j)}{i} .
$$

THEOREM 6.6. Let $w$ and $v$ be two different evolutions of the point $x_{0} \in T$. Then $\rho(w, v)=0$.

Proof. From a lemma of Weyl [47] it follows that the orbit of any point $x_{0} \in T$ is everywhere dense and evenly distributed. From the continuity of the map $f$, the condition $\operatorname{mes}(\partial U)=0$ and the definition of evolution it follows that $w_{n}=v_{n}$ if $f^{(n)}\left(x_{0}\right) \notin \partial U$. The proof of the theorem follows from these statements.

THEOREM 6.7. Let the points $x$ and $x^{*}$ be different and have different evolutions. Then the density of mismatch $\rho\left(w_{x}, w_{x^{*}}\right)$ is defined and is greater than 0 . 
Proof. We can assume that the words $w_{x}$ and $w_{x^{*}}$ differ in the first position. Consider the direct product $T \times T$. We divide the set of points into two classes: the first containing those words with the same current position and the second containing those words with different current position. Let $O \subset T \times T$ be the set of different pairs, then the orbit of the pair $\left(x, x^{*}\right)$ lies in $O$. The closed orbit of any pair is a torus, a minimal closed invariant set, on which the dynamics on the torus is realized. Let $\rho$ be the volume of the intersection, then $\rho \neq 0$ and $\rho$ is the density.

The mismatch function was introduced in [10] and studied by Yu. Pritykin [37.

6.6. Description of torus factor-dynamics. We will consider the dynamics that do not glue the coordinate $x_{n}$. Consider the $k$-th iteration of the transformation of the torus:

$$
\left\{\begin{aligned}
x_{m}^{(k)} & =x_{m}+C_{k}^{1} x_{m-1}+\ldots+C_{k}^{m} \varepsilon \bmod 1 \\
& \cdots \\
x_{i}^{(k)} & =x_{i}+C_{k}^{1} x_{i-1}+\ldots+C_{k}^{i} \varepsilon \bmod 1 \\
& \cdots \\
x_{1}^{(k)} & =x_{1}+C_{k}^{1} \varepsilon \bmod 1 .
\end{aligned}\right.
$$

If the points $A\left(x_{1}, \ldots, x_{m}\right)$ and $B\left(x_{1}+\Delta x_{1}, \ldots, x_{m}+\Delta x_{m}\right)$ belong to some quasiinvariant set $M^{\prime}$, then $f^{(k)}(A)$ and $f^{(k)}(B)$ also belong to the same set. From (3) it follows that

$$
\left\{\begin{aligned}
\Delta x_{m}^{(k)} & =\Delta x_{m}+C_{k}^{1} \Delta x_{m-1}+\ldots+C_{k}^{m-1} \Delta x_{1} \bmod 1, \\
& \ldots \\
\Delta x_{i}^{(k)} & =\Delta x_{i}+C_{k}^{1} \Delta x_{i-1}+\ldots+C_{k}^{i-1} \Delta x_{1} \bmod 1, \\
& \cdots \\
\Delta x_{1}^{(k)} & =\Delta x_{1} \bmod 1 .
\end{aligned}\right.
$$

Proposition 6.8. The closed orbit of any pair of points is a torus $T^{\prime}$, a minimal closed invariant set, on which the dynamics of the torus is realized.

REMARK 1. For the two-dimensional case, there exists an area $U$ with analytic boundary and a starting point $x^{*}$ that has infinitely many different evolutions, which differ in an infinite number of positions.

Proposition 6.9. Let $1, \varepsilon, \Delta x_{i}$ be linearly independent over $\mathbb{Q}$. Then the $A-B$ cloud contains all points whose first $i-1$ coordinates coincide with the corresponding coordinates of the point $B$.

Proof. We may assume that $\Delta x_{j}$ is rational when $j<i$ and let $\Delta x_{j}=p_{j} / q_{j}$ be the presentation of $\Delta x_{j}$ as an irreducible fraction and let $k$ be divisible by the product $m ! \prod_{j=1}^{i} q_{j}$, then $x_{j}^{(k l)}=x_{j}$ when $j<i$ and the systems 3 and 4 can be rewritten as 


$$
\left\{\begin{aligned}
x_{m}^{(k l)} & =x_{m}+C_{k l}^{1} x_{m-1}+\ldots+C_{k l}^{m} \varepsilon \bmod 1, \\
& \ldots \\
x_{j}^{(k l)} & =x_{j}+C_{k l}^{1} x_{j-1}+\ldots+C_{k l}^{j} \varepsilon \bmod 1, \\
& \ldots \\
x_{1}^{(k l)} & =x_{1}+C_{k l}^{1} \varepsilon \bmod 1 \\
\Delta x_{m}^{(k l)} & =\Delta x_{m}+C_{k l}^{1} \Delta x_{m-1}+\ldots+C_{k l}^{m-1} \Delta x_{1} \bmod 1, \\
& \cdots \\
\Delta x_{j}^{(k l)} & =\Delta x_{j}+C_{k l}^{1} \Delta x_{j-1}+\ldots+C_{k l}^{j-1} \Delta x_{1} \bmod 1 \quad(j \geq i), \\
\Delta x_{j}^{(k l)} & =\Delta x_{j} \bmod 1(j<i) .
\end{aligned}\right.
$$

The vector $\left(x_{1}^{(k l)}, \ldots, x_{m}^{(k l)}, \Delta x_{i}^{(k l)}, \ldots, \Delta x_{j}^{(k l)}\right)$ by the lemma of Weyl [47] is everywhere dense in the torus of dimension $2 m-i+1$, then from the definition of the $A-B$ cloud it follows that it contains all points whose first $i-1$ coordinates coincide with the corresponding coordinates of the point $B$, and the other points can be chosen arbitrarily.

Proposition 6.10. Let $\Delta x_{i}$ be an irrational number. Then there exists a point $B_{n}$, the evolution of which coincides with the evolution of the points $A$ and $B$, and for which

$$
\Delta x_{i}^{B_{n}}=n \Delta x_{i}^{B} .
$$

Proof. Let us choose points $A$ and $B$ as $B_{0}$ and $B_{1}$ respectively. By induction we will assume that the point $B_{k}$ is already built, and for $B_{k+1}$ it suffices to take any conditionally limit point of a sequence $f^{\left(n_{i}\right)}\left(B_{k}\right)$, under the condition $f^{\left(n_{i}\right)}\left(B_{k-1}\right) \rightarrow B_{k}$. Note that the point $B_{k}$ belongs to the $A-B k$-cloud.

Proposition 6.11. Let $\Delta x_{i}$ be an irrational number. Then there exists a point $B_{\delta}$, the evolution of which coincides with the evolutions of the points $A$ and $B$, and for which

$$
\Delta x_{i}^{B_{\delta}}=\delta, 0 \leq \delta \leq 1 .
$$

This fact follows directly from Proposition 6.10 and the fact that the set of points with fixed evolution is closed.

Thus the case when $\Delta x_{i}$ is irrational reduces to the case when $1, \varepsilon, \Delta x_{i}$ are linearly independent over $\mathbb{Q}$.

The case when all $\Delta x_{j}$ are rational leads to factor-dynamics where the $i$ edge of the torus divides into $M_{i}=\prod_{j=1}^{i} m_{j}$ parts, where $m_{j}$ are arbitrary natural numbers and the points of the torus $x=x^{*}$ are identified when for all $1 \leq j \leq m$ the equality $M_{j} x_{j}=M_{j} x_{j}^{*}$ holds.

The description of quasi-invariant sets follows from Proposition 6.11.

Theorem 6.12 (Description of quasi-invariant sets). A quasi-invariant set is a shift of an abelian group which transforms into themselves under translations by $1 / M_{i}$ along the $i$-coordinate or under all translations along $j$-coordinates with $j$ greater than some fixed number.

The theorem provides a description of all possible factor-dynamics. 
COROLlaRY 6.13. The class of closed reducible sets consists of sets, which transform into themselves under translations by $1 / M_{i}$ along the $i$-coordinate or under all translations along $j$-coordinates with $j$ greater than some fixed number. The set $0 \leq x_{m}<1 / 2$ is irreducible.

6.7. Proof of Theorem 1.1. This theorem follows from the next proposition:

Proposition 6.14. Consider the dynamics of the torus, given by equation (3) and let the irreducible set $U$ be given by the condition:

$$
0 \leq x_{m} \leq 1 / 2
$$

then there exists $L(\varepsilon)$ such that the points with the same finite evolution of length $L(\varepsilon) d i$ vide the torus into closed convex polyhedra, and different polyhedra correspond to different evolutions.

Proof. The set $U$ is irreducible, so by theorem 6.3 there exists a $\delta^{\prime}$ such that $N\left(\delta^{\prime}\right)$ evolutions of points at a distance greater than $\delta^{\prime}$ are different. It is obvious that $N$ evolutions of all interior points of the polyhedron obtained after $i$-th iteration are the same. We call it the evolution of the polyhedron.

Consider the parts of the partition, corresponding to words of length $N\left(\delta^{\prime}\right)$, then each polyhedron of the partition can intersect at most one hyperface from the family $n+1$ of planes $f^{n}\left[x_{1}=q / 2, q \in N\right.$, otherwise we can choose two points that belong to this polyhedron and are separated by two planes from the family $n+1$.

Assuming the family $n+1$ to be the first and turning the time back we get the situation when the $N\left(\delta^{\prime}\right)$ evolution of these points does not allow to distinguish them, and this is impossible. The polyhedra formed by the family $n+1$ of planes with the same evolution cannot have common points. Let $\delta^{*}$ be the minimal distance between the polyhedra with the same evolutions, then by putting $L(\varepsilon)=\max \left(N\left(\delta^{\prime}\right), N\left(\delta^{*}\right)\right)$ we obtain the number of iterations, from which certainly the equality between the number of words of length $k$ and the number of polyhedra into which the torus is divided by $k$ families of planes $f^{i}\left[x_{1}=q / 2, q=0, \ldots, k-1\right.$ is achieved. When $(\varepsilon)$ is irrational the intersection of more than $m$ hyperplanes is empty and there exists a one-to-one correspondence between the points of the intersection of $m$ non-parallel planes and the polyhedra of the partition. By computing the number of points in the intersection of $k$ families of planes $f^{i}\left[x_{1}=q / 2, q=0, \ldots, k-1\right.$ we obtain a polynomial, which defines the number of parts and consequently the number of subwords from some moment.

The number of points of intersection of the hyperplanes $Q(k)$ and consequently the number of subwords $T(k)(k \geq K)$ of length $k$ can be calculated by the formula:

$$
Q(k)=\sum_{0 \leq k_{1}<\ldots<k_{m} \leq k}\left|\begin{array}{cccc}
1 & \left(\begin{array}{c}
k_{m} \\
1
\end{array}\right) & \ldots & \left(\begin{array}{c}
k_{m} \\
m
\end{array}\right) \\
1 & \left(\begin{array}{c}
k_{1} \\
1
\end{array}\right) & \ldots & \left(\begin{array}{c}
k_{1} \\
m
\end{array}\right)
\end{array}\right|, \quad \operatorname{deg} Q(k)=\frac{m(m+1)}{2} .
$$

For every $K$ there exists some $P$ and $k_{0} \geqslant K$ such that the equality $T(k)=Q(k)$ holds for $k \geqslant k_{0}$ and does not hold for $k_{0}$. 


\section{References}

[1] A. Aberkane, Words whose complexity satisfies $\lim p(n) / n=1$, Theor. Comp. Sci. 307 (2003), 31-46.

[2] P. Arnoux, C. Mauduit, I. Shiokawa and J. Tamura, Complexity of sequences defined by billiard in the cube, Bull. Soc. Math. France 122 (1994), 1-12.

[3] P. Arnoux and C. Mauduit, Complexité de suites engendrées par des recurrences unipotentes, Acta Arith. 76 (1996), 85-97.

[4] P. Arnoux and G. Rauzy, Représentation géometrique des suites de complexité $2 n+1$, Bull. Soc. Math. France 119 (1991), 199-215.

[5] P. Baláži, Infinite words coding three-interval exchange, diploma work CTU (2003).

[6] P. Baláži, Substitution properties of ternary words coding 3-interval exchange, in: Proceedings WORDS 2003, T. Harju and J. Karhumaki (eds.), 2003, 119-124.

[7] P. Baláži, Z. Masáková and E. Pelantová, Characterization of substitution invariant words coding exchange of three intervals, Integers 8 (2008), No. 1, Article A20, 21 p.

[8] A. Ya. Belov, V. V. Borisenko, and V. N. Latyshev, Monomial Algebras, Itogi Nauki i Tekhniki, Sovr. Mat. Pril., Tem. Obzory, vol. 26 (Algebra IV), Moscow, 2002, 35-214 (Russian); Plenum, NY, 1998, 1-173 (English).

[9] A. Ya. Belov and A. L. Chernyatiev, Words with low complexity and interval exchange transformations, Uspehi Mat. Nauk 63 (2008), no 1, 159-160.

[10] A. Ya. Belov and G. V. Kondakov, Inverse problems of symbolic dynamics, Fundamental and Applied Mathematics 1 (1995), 71-79.

[11] J. Berstel, Recent results on Sturmian words, in: Developments in Language Theory II, World Scientific, 1996, 13-24.

[12] J. Berstel and P. Séébold, Sturmian words, in: Algebraic Combinatorics on Words, M. Lothaire (ed.), Encyclopedia of Mathematics and Its Applications 90, Cambridge University Press, Cambridge, 2002 (Chap. 2).

[13] V. Berthé, S. Ferenczi and L. Q. Zamboni, Interactions between dynamics, arithmetics and combinatorics: the good, the bad, and the ugly, in: Algebraic and Topological Dynamics, Contemporary Mathematics, AMS, 2007, 333-364.

[14] A. I. Bufetov, Finitely-additive measures on the asymptotic foliations of a Markov compactum, http://arxiv.org/abs/0902.3303v1.

[15] J. Cassaigne, Special factors with linear subword complexity, in: Developments in Language Theory, II (Magdeburg, 1995), World Sci. Publ., River Edge, NJ, 1996, 25-34.

[16] J. Cassaigne, P. Hubert and S. Troubetzkoy, Complexity and growth for polygonal billiards, Ann. Inst. Fourier (Grenoble) 52 (2002), 835-847.

[17] A. L. Chernyatiev, Balanced Words and Dynamical Systems, Fundamental and Applied Mathematics 13 (2007), 213-224.

[18] A. L. Chernyatiev, Words with minimal growth function, Vestnik Mosk. Gos. Univ., 2008.

[19] X. Droubay, J. Justin and G. Pirillo, Episturmian words and some constructions of de Luca and Rauzy, Theoret. Comp. Sci. 255 (2001), 539-553.

[20] S. Ferenczi, C. Holton and L. Q. Zamboni, Structure of three-interval exchange transformations II: a combinatorial description of the trajectories, J. Analyse Math. 89 (2003), 239-276.

[21] S. Ferenczi and L. Q. Zamboni, Combinatorial structure of symmetric $k$-interval exchange transformations, preprint. 
[22] S. Ferenczi and L. Zamboni, Languages of $k$-interval exchange transformations, Bull. Lond. Math. Soc. 40 (2008), 705-714.

[23] H. Furstenberg, Poincaré recurrence and number theory, Bull. Amer. Math. Soc. 5 (1981), 211-234.

[24] R. L. Graham, Covering the positive integers by disjoint sets of the form $\{[n \alpha+\beta]: n=$ $1,2, \ldots\}$, J. Combin. Theory Ser. A15 (1973), 354-358.

[25] R. H. Herman, I. F. Putnam and C. F. Skau, Ordered Bratteli diagrams, dimension groups and topological dynamics, Internat. J. Math. 3 (1992), 827-864.

[26] P. Hubert, Well-balanced sequences, Theoret. Comput. Sci. 242 (2000), 91-108.

[27] R. N. Izmailov and A. A. Vladimirov, Dimension of aliasing structures, Journal of Applied Mathematics and Stochastic Analysis, to appear.

[28] A. Ya. Kanel-Belov and A. L. Chernyatiev, Describing the set of words generated by interval exchange transformation, Comm. in Algebra 38 (2010), 2588-2605.

[29] A. Kanel-Belov and I. Mitrofanov, Periodicity of Rauzy scheme and substitutional systems, to appear; http://arxiv.org/abs/1107.0185

[30] L. Kuipers and H. Niederreiter, Uniform Distribution of Sequences, John Wiley \& Sons, New York, 1974.

[31] A. N. Livshits, Application of adic representations in the investigations of metric, spectral and topological properties of dynamical systems, Thesis, Sankt-Petersburg, 1995.

[32] M. Lothaire, Combinatorics on Words, Encyclopedia of Mathematics and its Applications 17, Addison-Wesley, Reading, MA, 1983.

[33] A. de Luca, Sturmian words: structure, combinatorics and their arithmetics, Theoret. Comp. Sci. 183 (1997), 45-82.

[34] C. Mauduit, Substitutions, arithmetic and finite automata: an introduction, in: Substitutions in Dynamics, Arithmetics and Combinatorics, Lecture Notes in Math. 1794, Springer, Berlin, 2002, 35-52.

[35] M. Morse and G. A. Hedlund, Symbolic dynamics II. Sturmian trajectories, Amer. J. Math. 62 (1940), 1-42.

[36] An. A. Muchnik, Yu. L. Pritykin and A. L. Semenov, Sequences close to periodic, Uspekhi Mat. Nauk 64 (2009), no. 5, 21-96 (in Russian).

[37] Y. Pritykin and J. Ulyashkina, A periodicity Measure for Infinite Sequences, in: Proceedings of CSR 2009, Novosibirsk, Lecture Notes in Computer Science 5675, Springer, 2009, 274-285,

[38] L. D. Pustylnikov, Distribution of the fractional parts of a polynomial, Weyl sums, and ergodic theory, Uspekhi Mat. Nauk 48 (1993), no. 4, 131-166.

[39] G. Rauzy, Echanges d'intervalles et transformations induites, Acta Arith. 34 (1979), 315328.

[40] G. Rauzy, Nombres algébriques et substitutions, Bull. Soc. Math. France 110 (1982), 147178.

[41] G. Rauzy, Mots infinis en arithmétique, in: Automata on Infinite Words (Le Mont Dore, 1984), M. Nivat and D. Perrin (eds.), Lecture Notes in Computer Science 192, Springer, Berlin, 1985, 165-171.

[42] G. Rote, Sequences with subword complexity 2n, J. Number Theory 46 (1994) 196-213.

[43] Ya. G. Sinai, Introduction to Ergodic Theory, Princeton Univ. Press, Princeton, NJ, 1977; Moscow, Fazis, 1996.

[44] A. M. Vershik, The adic realizations of the ergodic actions with the homeomorphisms of the Markov compact and the ordered Bratteli diagrams, Zap. Nauchn. Sem. S.-Peterburg. Otdel. Mat. Inst. Steklov. (POMI) 223 (1995), 120-126, 338; translation in J. Math. Sci. (New York) 87 (1997), 4054-4058. 
[45] A. M. Vershik and A. N. Livshits, Adic models of ergodic transformations, spectral theory, substitutions, and related topics, in: Representation Theory and Dynamical Systems, Adv. Soviet Math. 9, Amer. Math. Soc., Providence, RI, 1992, 185-204.

[46] L. Vuillon, Balanced words, Bull. Belg. Math. Soc. Simon Stevin 10 (2003), 787-805.

[47] H. Weyl, Über die Gleichverteilung von Zahlen mod. 1, Math. Ann. 77 (1916), 313-352. 